\title{
BMJ Open Dietary phytochemical intake from foods and health outcomes: a systematic review protocol and preliminary scoping
}

\author{
Yasmine C Probst, ${ }^{1,2}$ Vivienne X Guan, ${ }^{1,2}$ Katherine Kent ${ }^{1,3}$
}

To cite: Probst YC, Guan VX, Kent K. Dietary phytochemical intake from foods and health outcomes: a systematic review protocol and preliminary scoping. BMJ Open 2017;7:e013337. doi:10.1136/bmjopen-2016013337

- Prepublication history and additional material is available. To view please visit the journal (http://dx.doi.org/ 10.1136/bmjopen-2016013337)

Received 7 July 2016 Revised 8 December 2016 Accepted 12 December 2016

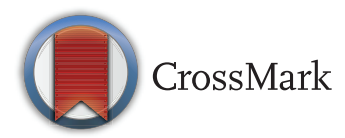

${ }^{1}$ Faculty of Science, Medicine and Health, University of Wollongong, Wollongong, New South Wales, Australia ${ }^{2}$ Illawarra Health and Medical Research Institute, Wollongong, New South Wales, Australia

${ }^{3}$ Faculty of Health, University of Tasmania, Tasmania, Australia

Correspondence to Dr Yasmine C Probst; yasmine@uow.edu.au

\section{ABSTRACT}

Introduction: Dietary phytochemicals are found in plant-based foods such as fruits, vegetables and grains and may be categorised in a nested hierarchical manner with many hundred individual phytochemicals identified to date. To associate phytochemical intakes with positive health outcomes, a fundamental step is to accurately estimate the dietary phytochemical intake from foods reported. The purpose of this systematic review protocol is to describe the process to be undertaken to summarise the evidence for food-based dietary phytochemical intakes and health outcomes for adults.

Methods and analysis: The review will be undertaken following the PRISMA guidelines and the Cochrane Handbook for Systematic Reviews of Interventions using the Review Manager software. Phytochemical subclasses (phenolic acids, flavanols, etc) will be used to search for relevant studies using the Web of Science and Scopus scientific databases. The retrieved studies will be screened based on inclusion of natural whole food items and health outcomes. Phytochemical studies related to cardiovascular disease, cancer, overweight, glucose tolerance, digestive, reproductive, macular and bone health and mental disorders, fatigue and immunity will be examined based on prior scoping. The evidence will be aggregated by the food types and health outcomes. Comparison of differences in the outcomes for randomised controlled trials and observational studies will be undertaken.

The strength of the review lies in its focus on whole food items and health conditions rather than one type of phytochemical related to one single health condition. Subgroup and sensitivity analyses will be conducted where an adequate number of publications are found per phytochemical subclass.

Dissemination: By comparing the outcomes from experimental and observational studies, the review will determine whether the overall conclusions related to the phytochemical subclasses are the same between study types for the identified health conditions. This is useful to public health policymakers and health professionals alike. Trial registration number: \#CRD42014015610.

\section{BACKGROUND}

Dietary phytochemicals are commonly found in plant-based foods such as fruits, vegetables, grains and tea. ${ }^{1}$ Consumption of total phytochemical intake is consistently linked with protection from chronic diseases, ${ }^{2}$ including cardiovascular disease, ${ }^{3-5}$ cancer $^{67}$ and neurodegenerative diseases. ${ }^{8}$ In order to associate phytochemical intakes from food with positive health outcomes, a fundamental step is to accurately estimate dietary phytochemical intakes. Despite the first estimations of phytochemical intake from food at a population level being reported more than a decade ago, the methods currently employed have evident flaws. ${ }^{9}$ Traditional methods of dietary assessment require a recall or documentation of food intakes from a given time period in either a prospective or retrospective manner. ${ }^{10}$ To determine the nutrient composition of individual or group intakes, this dietary intake data needs to be converted to an estimated consumption of the 'nutrient' of interest. This can be performed by applying a phytochemical food composition database (FCDB) to the dietary intake data. ${ }^{11}$

Aside from the limitations associated with each dietary assessment method, ${ }^{10}$ there are also several well-documented problems associated with using FCBDs to assign phytochemical content (including type and concentration) to selected foods, resulting in large variations in estimates of intake. ${ }^{9} 12$ First, estimation of dietary phytochemical intake is only as comprehensive as the FCDB used. If, for example, a database does not have an extensive list of food items and the phytochemical content of a food in an individual's diet cannot be assigned or matched to its closest equivalent, ${ }^{13}$ then an individual's intake will be underestimated. This is particularly challenging when analysing food intake 
data from a country that does not have a phytochemical FCDB specific to the food supply, resulting in significant sources of phytochemicals not being captured. ${ }^{14}$ The primary FCDBs used by studies (USDA, Phenol Explorer and Euro FIR-eBASIS) provide extensive information on the individual phytochemicals in foods, with additional details provided for the variability of phytochemical contents in foods, as well as the values associated with processing. It is interesting to note that using different FCDB may substantially influence the estimation of phytochemical intake in a study, as highlighted by a recent comparison between estimates of dietary phytochemical intake produced by the US Department of Agriculture (USDA) and Phenol Explorer databases. ${ }^{15}$ However, this is not a well-researched concept and needs further verification. Second, the phytochemical content of specific foods is highly variable and influenced by a foods growth, harvest and processing conditions. Disregarding the processes by which foods are produced and the impact on the phytochemical levels may lead to an overestimation of the phytochemical intakes. A phytochemical FCBD is unable to account for all of the variability and can only provide an estimate for each food consumed. Finally, estimating dietary phytochemical intakes through dietary assessment is unable to account for the high intraindividual variation associated with phytochemical metabolism and absorption, which is influenced by factors other than intake, such as bioavailability and genetic factors.

\section{Rationale}

\section{Description of the problem or issue}

Dietary phytochemicals are bioactive compounds that are commonly found in plant-based foods such as fruits, vegetables, grains and tea. Many studies, including animal models, population observations and clinical trials, have been conducted to investigate the protective effects of dietary phytochemical intakes from food. ${ }^{16-21}$

Phytochemicals may be categorised in a nested hierarchical manner of classes and subclasses. For example, total polyphenols comprises flavonoids, phenolic acids, lignans and other polyphenol groups. ${ }^{22}$ Flavonoids can be further divided into anthocyanins, flavan-3-ols, flavanones, flavones, flavonols, isoflavones and proanthocyanidins subclasses. ${ }^{22}$ Current published reviews tend to focus only on the relationship between a single subclass of phytochemical and one health condition regardless of the source of phytochemical, that is, compound extraction and synthesis, or from whole food source. ${ }^{23-28}$ Although it is valuable to isolate the specific phytochemical to reveal their mechanism of action, it may be problematic in reflecting dietary consumption. People eat food and therefore dietary phytochemical research should consider the whole food matrix.

Many whole foods, such as apples, blueberries, broccoli, cherries, soy beans and walnuts, are naturally rich in phytochemicals, but are also high in micronutrients and fibres. ${ }^{29}{ }^{30}$ As phytochemical compounds do not exist in isolation in these foods, the whole food matrix may play a role in the protective effects of phytochemicals. It has been suggested that phytochemicals consumed as a part of their natural food matrix may be more bioactive than a high dose of isolated phytochemicals provided by dietary supplements. ${ }^{31}$ This may be attributed to phytochemicals working synergistically with other phytochemicals that are inherently present in the food ${ }^{32}$ Furthermore, their bioactivity may also be attributable to the interactions between the phytochemicals and other nutrients (eg, vitamin $\mathrm{C}$ ) ${ }^{31}$ or other constituents or contaminants in the food. For example, polyphenols found in whole grain foods have been considered to contribute to the health benefits observed. ${ }^{33}$ However, the health benefits of whole grain consumption related to polyphenols may also be linked to the cereal fibre or other components that naturally occurs in the grain. ${ }^{34}$ Such patterns cannot be determined by isolating the phytochemicals. Reviews have been conducted previously for specific phytochemical-rich whole food items such as strawberries; ${ }^{35}$ however, the focus remained on single phytochemical subclasses, namely, strawberry polyphenols. Such reviews should consider the synergistic interactions occurring within the phytochemical-rich whole food matrix. Although other nutrients, such as micronutrients and fibre, naturally occur in phytochemical-rich whole foods, the phytochemical content of a given whole food can be estimated using dietary assessment tool and phytochemical FCDBs, which can be further compared across the studies. Thus, grouping and analysing phytochemical content of phytochemical-rich whole foods can still be achieved, but may also need to take into account the foods synergistic effect and human consumption habits. Examining phytochemical-rich whole foods rather than specific classes of phytochemicals may reflect the dietary consumption characteristics that are in turn translatable to dietary advice.

Translation to dietary advice also requires consideration of the health conditions being addressed. Common risk factors and biomarkers have been found for different diseases, particularly lifestyle-related chronic diseases such as cardiovascular disease, type 2 diabetes mellitus and various forms of cancer. Endothelial dysfunction biomarkers including E-selectin, intercellular adhesion molecule 1 and vascular cell adhesion molecule 1 are common risk factors related to cardiovascular diseases and type 2 diabetes mellitus, ${ }^{36}{ }^{37}$ while inflammatory biomarkers such as C-reactive protein are common to the development of type 2 diabetes mellitus and cancer. ${ }^{38} 39$ Therefore, this review will consider a range of whole food items and disease states, which to date have not been addressed in reviews of this nature.

Another limitation hindering the interpretation of phytochemical studies are the differences between observational and experimental research outcomes that demonstrate associations of dietary phytochemical intakes with specific health outcomes. ${ }^{5}{ }^{40}$ Population- 
based observational studies often provide evidence of associations between diet and disease, while experimental studies, particularly randomised controlled trials, are designed to reduce bias and confounding to reveal relationships via hypothesis testing of outcomes from these observational studies. ${ }^{41}$ A comparison of outcomes related to phytochemical intakes in observational and experimental research is imperative to interpret current findings across the literature and to provide recommendations for future research.

\section{Description of the methods being investigated}

Figure 1 provides an overview of the proposed approach to the systematic literature review to be conducted. There are currently numerous methods used to measure dietary phytochemical intakes in different settings. This systematic review will consider studies that use any form of dietary assessment translated to 'nutrient' data to estimate consumption of dietary phytochemicals or the intake of specific phytochemical-rich food items. Although it is recognised that biomarkers of intake may be used as a means to measure intake, studies using biomarkers of intake will not be included in the review. Validation and methodological studies for dietary assessment tools will also be excluded as the outcomes are unlikely to relate to the health outcomes, the focus of the review.

\section{How these methods might work}

The proposed review will consider the various subclasses of the phytochemical class overall. Although the evidence aggregation will be based on individual food items, it will specifically focus on the whole food matrix and its nutrient synergies. For example, foods high in whole grains are also high in dietary fibre and polyphenols, a class of phytochemical. For this review, the interest is related to the polyphenols content of the wholegrain foods rather than the dietary fibre. Therefore, the specific phytochemical compounds will be used to search for the relevant studies to capture the various subclasses of the phytochemical class overall. As common risk factors and health biomarkers are shared across different health conditions, as demonstrated

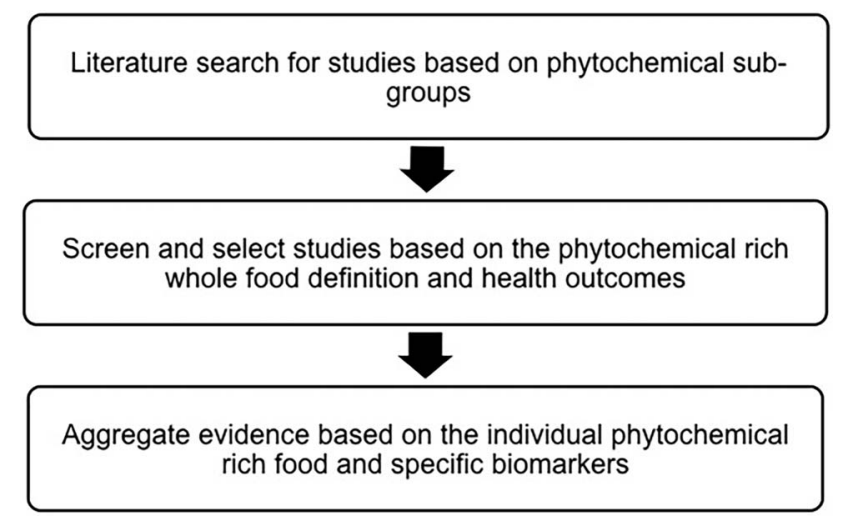

Figure 1 Overview of the proposed approach to the systematic literature review to be conducted. above, the health outcomes will not be limited during the key word searches. The health outcomes-related phytochemical studies will be identified during the study abstract screening phase. In the evidence synthesis phase, the phytochemical content of phytochemical-rich whole foods will be extracted and summarised based on the reported values and units. The identified values will be used as a benchmark to compare across studies. Finally, aggregation of evidence based on the individual foods rather than the phytochemical classes is more likely to reflect eating behaviours, as different phytochemical compounds may have positive protective effects on the same biomarker or health outcome. For example, brassica vegetables such as broccoli, Brussels sprouts and cabbage are naturally low in flavonoid compounds though are high in glucosinolates. ${ }^{42}$ Flavonoids and glucosinolates are phytochemicals and have demonstrated protective effects related to cancer risk in human population studies. ${ }^{43} 44$ Thus, the proposed approach to the systematic literature review will provide novel insight into health outcomes associated with phytochemical-rich food consumption, while considering eating behaviours. Within the studies, the methods used to determine the phytochemical content of the food items will be considered to determine if there is consistency within the health outcome categories of interest.

\section{Why it is important to do this review}

As stated earlier, current reviews with phytochemicals focus on one health condition and/or one specific subclass of phytochemical, for example, anthocyanins. Further to this, other reviews focus on either experimental or observational study designs, but do not attempt to compare both. ${ }^{45-47}$ Although the workload substantially increases when considering all studies, this will allow the current review to determine whether the conclusions being made by the differing study types are consistent. In addition, focussing on phytochemicals from whole food sources rather than from a process of compound extraction and/or synthesis again relates to eating behaviours. By considering a range of phytochemical subclasses and health outcomes, this review will relate outcomes to the whole food matrix of different foods rather than focusing on a single specific food components which to date have also not been addressed.

\section{Objectives}

The purpose of this review is to summarise and synthesise the evidence about dietary phytochemical intakes from whole foods related to health outcomes in randomised and non-randomised food-based trials, prospective and retrospective cohort, case-control, cross-sectional studies that aim to measure or estimate dietary phytochemical intakes in adults. The specific question that the review following this protocol will address utilises a PICO format and is proposed as: In food-based studies for phytochemical intake, what is the impact on chronic disease outcomes for adults? 
The following questions will also be used guide the literature search:

- What is the primary health outcome reported from the study and how does this relate to chronic disease?

- Does the study focus on whole foods, not supplements?

- Are the phytochemical outcomes a primary focus to the 'nutrients' reported for the study?

It is hypothesised that the review will show an association between the number of studies related to a phytochemical subclass and consistency of the outcomes reported.

\section{METHODS}

This review was registered with the International Prospective Register of Systematic Reviews (PROSPERO) under the registration number \#CRD42014015610.

The structure of this review protocol follows the Preferred Reporting Items for Systematic Review and Meta-Analyses (PRISMA) guidelines and the recommendations of the Cochrane Handbook for Systematic Reviews of Interventions using Review Manager (RevMan) Computer program, V.5.3.5, Copenhagen: The Nordic Cochrane Centre, The Cochrane Collaboration, 2014. This protocol was developed in line with the PRISMA-P 2015 checklist. ${ }^{48} 49$

\section{Eligibility criteria for considering studies \\ Types of studies}

This review will include randomised, non-randomised food-based trials and crossover food-based trials, and analytical epidemiological study designs including prospective and retrospective cohort, case-control and cross-sectional studies.

\section{Types of participants}

No restrictions will be set for the age or gender of participants, though participants must be diagnosed with a chronic disease. These chronic diseases will be used for subgroup statistical analyses.

\section{Setting and time frame}

Studies will be restricted to those published from January 2004 to March 2016 to ensure the literature is relevant. Studies published before 2004 are not included due to a focus on mechanistic studies as identified during the scoping phase.

\section{Types of data}

The main data will be the dietary phytochemical intakes from foods related to health outcomes from different study designs. The details of study characteristics and consumption of phytochemicals will be examined. The trends and patterns of dietary phytochemical intake from foods and health outcomes in different study designs will be explored.

\section{Types of methods}

Studies reporting data for whole food-based phytochemical outcomes in relation to a health condition will be included. Studies that do not measure whole foods or whole of diet-based phytochemical intakes (ie, encapsulated phytochemicals extract, extract from herbal sources and purified or modified version of phytochemicals) and other mechanistic studies (ie, bioavailability or mechanistic feeding studies) will be excluded. The phytochemicalcontaining foods must be identified as naturally containing phytochemicals (with a reference to a study characterising the phytochemical composition of the food, or reference to a comprehensive FCDB). The identified phytochemical-rich food must also be commercially available or publicly accessible by a general population.

\section{Information sources}

The search aims to find published and unpublished studies through electronic scientific databases, the Internet and reference lists of included studies. Key terms have been developed (see online supplementary appendix 1). The initial phase will consist of searches of the following databases as the two largest health-related databases:

- Web of Science

- Scopus

Only studies published in the English language will be considered for inclusion due to a lack of translation resources. Ongoing clinical trials will be searched for using the Australian Government National Health and Medical Research Council and Department of Industry, Innovation and Science Clinical trials registries (https:// www.australianclinicaltrials.gov.au/clinical-trial-registries) using the phytochemical subclasses listed in online supplementary appendix 1 .

\section{Search strategy}

An example search strategy as applied to the Scopus scientific database is shown below including limits to the year of publication, document type and a focus on human studies to exclude many of the mechanistic studies.

(TITLE-ABS-KEY (lignans OR 'flavan-3-ol*' OR terpene* OR carotenoid* OR limonoid* OR phytosterol* OR thiol* OR glucosinolate* OR indole* OR isoprenoid* OR 'Lipoic acid' OR ubiquinone OR 'Phenolic acid' OR flavonoid* OR flavanol* OR tannin* OR stilbene* OR lignans OR isoflavone* OR anthocyanin* OR flavanone* OR flavonol* OR flavone* OR allicin OR capsaicin OR catechin OR 'Ellagic acid' OR genistein OR lycopene OR saponin* OR zeaxanthin OR polyphenol*) AND TITLE-ABS-KEY (((randomi?ed W/6 control) AND (trial OR study)) OR (cohort W/6 stud*) OR (longitudinal W/6 stud*) OR 'panel stud*') AND NOT TITLE-ABS-KEY (rat OR mice OR cell)) AND PUBYEAR > 2004 AND (LIMIT-TO (DOCTYPE, 'ar') OR LIMIT-TO (DOCTYPE, 're') OR LIMIT-TO (DOCTYPE, 'ip')) AND (LIMIT-TO (EXACTKEYWORD, 'Human') OR LIMIT-TO (EXACTKEYWORD, 'Humans'))

\section{Study records}

\section{Selection process}

The review will be structured and reported according to PRISMA. One review author (YCP) will conduct the literature search in the specified scientific databases. Results of 
the search will be collated in the reference management software Endnote (version X7.5, Thomson Reuters, 2016). Duplicate publications will be excluded. Two review authors (KK and VXG) will independently screen publications using the criteria (table 1) for inclusion. Resolution of any disagreements will occur through discussion and required consultation with a third reviewer (YCP) for consensus.

The observational studies will be considered separately to other studies to determine any differences in methods reported. Articles identified by database searches will be assessed for relevance to the review based on the title and abstract (table 1). For those meeting the inclusion criteria, the full-text publications will be retrieved and assessed for relevance to the review criteria. A PRISMA flow diagram of the search strategy and selection process will be developed.

\section{Data extraction and data management}

Eligibility for inclusion will be confirmed by an independent researcher (SM). Data extracted from the studies will be entered into RevMan software V.5.3.5 by the same researcher. Data accuracy will be checked by double data entry having one review author (VXG) entering data into a separate file and comparing the results for a subset of studies. Consensus will be sought by consultation with a third researcher (YCP). When the information regarding any of the above is unclear, YCP will attempt to contact authors of the original publication to provide further details. The studies will be grouped, described and evaluated in accordance with their methodological similarities. Studies will also be separated into their experimental or observational design. Studies will be further grouped by their health condition and phytochemical subclass.

\section{Data collection process}

To ensure internal bias is minimised, the review will be undertaken by more than one researcher with quality assessed between the methods of data extraction.

\section{Outcome measures and prioritisation \\ Primary outcomes}

The primary outcome is the summary of dietary phytochemical intakes from foods by subclass and health outcomes from different study designs. The phytochemical subclasses were determined based on the classification system outlined in the Handbook of plant food phytochemicals. ${ }^{22}$

This review will consider studies that investigate dietary phytochemical intake from whole foods by addressing at least one of the following health outcomes:

Health outcomes:

- Cardiovascular disease

- Cholesterol

- Heart disease

- Hypertension

- Blood pressure

- Cancer

- Oxidative stress

- DNA damage

- Overweight

- Obesity

- Weight loss

- Body composition

- Glucose tolerance

- Diabetes mellitus (type 1 and 2)

- Metabolic syndrome

- Digestion

- Gastrointestinal tract

- Constipation

Data items

Table 1 Overall inclusion and exclusion criteria for publication screening

\begin{tabular}{|c|c|c|}
\hline Section & Criteria & $\begin{array}{l}\text { Include } \\
\text { if: }\end{array}$ \\
\hline Language & Publication reported in English & Yes \\
\hline \multirow[t]{2}{*}{ Design } & $\begin{array}{l}\text { Randomised or non-randomised food-based trials, or prospective or retrospective cohort or, case- } \\
\text { control, cross-sectional studies }\end{array}$ & Yes \\
\hline & Case reports, reviews, editorials, letter to the editor, qualitative research & No \\
\hline \multirow[t]{2}{*}{ Population } & Adults aged $18+$ years & Yes \\
\hline & Animal studies or studies including $<18$ years & No \\
\hline \multirow[t]{6}{*}{ Content } & - Studies examining the relationship between total or specific dietary phytochemical intake from & Yes \\
\hline & $\begin{array}{l}\text { The intervention advises participants to consume foods rich in phytochemicals: } \\
\text { - Natural whole food, include powder and puree which are food composition complete, such as } \\
\text { blueberries and apple }\end{array}$ & $\begin{array}{l}\text { Yes } \\
\text { No }\end{array}$ \\
\hline & - Natural whole food juice or drink, such as apple juice & No \\
\hline & $\begin{array}{l}\text { - Complex food, which contains more than one whole food ingredients combination, for example, } \\
\text { fortified bread or cereal bar }\end{array}$ & \\
\hline & $\begin{array}{l}\text { Studies examining encapsulated phytochemicals, extract from herbal sources and purified or } \\
\text { modified version of phytochemicals. }\end{array}$ & \\
\hline & Mechanistic studies (ie, bioavailability or mechanistic feeding studies) & \\
\hline Access & Full-text article accessible & Yes \\
\hline
\end{tabular}


- Reproductive health

- Macular health

- Bone health

- Mental health

- Cognitive processes

- Cognition

- Mood

- Attention

- Memory

- Fatigue

- Immunity

The health outcomes are based on preliminary scoping searches in relation to the topic area being proposed (table 2). Owing to the variability of the health conditions, the specific outcomes of interest will be targeted based on consistency of reporting within the studies for a health condition and in turn for a phytochemical subclass. The authors will consider the requirement for a three-part review series capturing cardiovascular disease, cancer and other health conditions where consistency is apparent. This will be determined following application of the inclusion criteria to the publications retrieved from the database search. It is likely that common factors such as height, weight, body mass index, gender and mortality (as appropriate) can be applied across all conditions and the principles of the protocol applicable to a series or single review.

\section{Assessment of reporting biases in individual studies}

Systematic error as a result of bias will be considered to account for selection bias as well as any reporting bias that may arise within the review.

Two review authors (KK and VXG) will independently assess the quality for each study using the Cochrane Risk of Bias Tool $^{50}$ to address the various forms of bias related to the extracted studies. This tool will be used to critically appraise the quality of included studies, looking at issues of validity of included studies such as relevance to improve current practice, randomisation, allocation concealment, blinding, intervention description, validity and reliability of measurements, missing data, selective reporting. Studies will be scored as low, unclear or high according to the specified criteria. Studies will not be excluded on the grounds of their quality, but the review will report methodological quality when presenting the results.

\section{Data synthesis}

All results will be subject to a rigorous quality assessment process to ensure data quality is upheld. For all aims, findings will be presented as a narrative synthesis, as well as tables and figures. Data synthesis and required statistical analyses as outlined below will be conducted by YCP and VXG.

\section{Dealing with missing data}

Where key data are missing from the included publications, the review authors will attempt to contact corresponding authors (or other authors if necessary) of included studies. If this information is not achievable, the data will not be imputed and a note will be made that the study did not provide data for the particular outcome.

\section{Sensitivity analysis}

Although it is unlikely that a meta-analysis will be conducted with the data extracted, sensitivity will be considered to ensure the decision-making process was robust. It will demonstrate the influence of the abovementioned potentially missing data with and without imputations on the findings of the review. Further to this during the review process, abstracts that do not contain sufficient data will be included rather than excluded and progress to a second review phase of full-text publications. Studies will only be included if all participants of the study meet the inclusion criteria. Time points at which tools in a study are used, for example, dietary assessment methods will also be extracted for comparability of data trends.

\section{Subgroup analysis and investigation of heterogeneity}

Included publications will be grouped by their phytochemical subclass to determine patterns of reporting in relation to assessment tools used. The weighted mean difference of the intervention and control groups for randomised controlled trial studies will be calculated and presented with $95 \%$ CIs. If mean values are not provided in the publication, it will be computed using CIs and the SEM. Heterogeneity of the participant population data will be confirmed using a random effects model. Any abnormal data points will be checked to ensure incorrect data have not been reported. $\chi^{2}$ and $\mathrm{I}^{2}$ tests will be conducted to determine the level of heterogeneity. The $95 \%$ CI of the $\mathrm{I}^{2}$ will be presented and $75 \%$ will be identified as considerable heterogeneity. ${ }^{50}$ High variability of the data is expected.

\section{Meta-bias(es)}

Bias related to the systematic error, selective reporting or publication bias will be considered for all studies included in the review. This will be determined using Funnel plots. The scale reversed SE plotted against the effect estimate with $95 \%$ CIs will be considered to address small sample size bias. This will be indicative of an asymmetrical plot where the log of the OR to be considered.

\section{Confidence in cumulative evidence}

The strength of the body of evidence will be assessed for each health outcome addressed in the review using the Grading of Recommendations Assessment, Development and Evaluation (GRADE) instrument. ${ }^{50}$ This includes consideration of the study limitations, indirectness, inconsistency, imprecision, publication bias within the studies related to the health outcomes. 
Table 2 Preliminary scoping of publications grouped by phytochemical subclass

\begin{tabular}{|c|c|c|c|c|c|c|c|c|c|c|c|}
\hline \multirow[b]{2}{*}{ Subclass ${ }^{21}$} & \multirow[b]{2}{*}{$\begin{array}{c}\text { Total } \\
\text { publications }\end{array}$} & \multirow[b]{2}{*}{ Biomarker } & \multirow[b]{2}{*}{ CVD } & \multirow[b]{2}{*}{$\begin{array}{l}\text { Cancer } \\
\text { protection }\end{array}$} & \multirow[b]{2}{*}{$\begin{array}{l}\text { Cancer } \\
\text { care }\end{array}$} & \multirow[b]{2}{*}{$\begin{array}{l}\text { Mental } \\
\text { health }\end{array}$} & \multirow[b]{2}{*}{$\begin{array}{l}\text { Other } \\
\text { disease }\end{array}$} & \multicolumn{2}{|l|}{ Review } & \multirow[b]{2}{*}{$\begin{array}{c}\text { Purified } \\
\text { component }\end{array}$} & \multirow[b]{2}{*}{$\begin{array}{c}\text { Total health } \\
\text { outcomes }\end{array}$} \\
\hline & & & & & & & & Food-related & Non-food-related & & \\
\hline Phenolic acids & 138 & 20 & 15 & 15 & 1 & 2 & 8 & 16 & 42 & 9 & 46 \\
\hline Flavonoids & 678 & 21 & 104 & 24 & 41 & 14 & 45 & 84 & 283 & 22 & 260 \\
\hline Flavanols & 26 & 0 & 13 & 1 & 1 & 3 & 1 & 3 & 4 & 0 & 19 \\
\hline Tannins & 172 & 0 & 4 & 0 & 0 & 0 & 5 & 0 & 163 & 0 & 9 \\
\hline Stilbenes & 63 & 3 & 0 & 0 & 0 & 0 & 3 & 7 & 49 & 0 & 3 \\
\hline Lignanss & 133 & 14 & 13 & 1 & 22 & 3 & 23 & 10 & 28 & 6 & 67 \\
\hline Isoflavones & 706 & 44 & 28 & 12 & 56 & 3 & 75 & 71 & 209 & 9 & 362 \\
\hline Anthocyanins & 49 & 1 & 4 & 3 & 0 & 0 & 6 & 4 & 25 & 4 & 13 \\
\hline Flavanones & 11 & 2 & 0 & 0 & 0 & 0 & 0 & 0 & 9 & 0 & 0 \\
\hline Flavonols & 28 & 0 & 1 & 3 & 1 & 1 & 1 & 7 & 15 & 0 & 7 \\
\hline Flavones & 14 & 0 & 0 & 0 & 0 & 0 & 0 & 1 & 13 & 0 & 0 \\
\hline Allicins & 16 & 0 & 0 & 0 & 0 & 0 & 0 & 5 & 6 & 0 & 5 \\
\hline Capsaicins & 609 & 0 & 1 & 1 & 0 & 0 & 13 & 3 & 590 & 1 & 15 \\
\hline Catechins & 143 & 2 & 5 & 3 & 3 & 0 & 11 & 14 & 105 & 0 & 22 \\
\hline Ellagic acids & 12 & 0 & 0 & 0 & 0 & 0 & 0 & 0 & 12 & 0 & 0 \\
\hline Genisteins & 0 & 0 & 0 & 0 & 0 & 0 & 0 & 0 & 0 & 0 & 0 \\
\hline Lycopenes & 116 & 4 & 8 & 8 & 17 & 0 & 10 & 7 & 56 & 0 & 43 \\
\hline Saponins & 72 & 1 & 0 & 0 & 0 & 0 & 0 & 0 & 70 & 1 & 0 \\
\hline Zeaxanthins & 73 & 3 & 0 & 1 & 1 & 0 & 13 & 5 & 50 & 0 & 15 \\
\hline Polyphenols & 244 & 8 & 40 & 13 & 17 & 3 & 26 & 30 & 98 & 8 & 99 \\
\hline Flavan-3-ols & 66 & 2 & 13 & 4 & 12 & 9 & 6 & 6 & 6 & 4 & 44 \\
\hline Total & 3369 & 125 & 249 & 89 & 172 & 38 & 246 & 273 & 1833 & 64 & 1029 \\
\hline
\end{tabular}

3369

*Where summation of the row

CVD, cardiovascular disease. 


\section{Management and review dissemination}

The processes of review and synthesis outlined in this protocol will be undertaken by at least two members of the review team to ensure quality assurance throughout the review. Management of the review team will be upheld by YCP. The entire review team will meet following completion of each stage of the review. By comparing the outcomes from experimental and observational studies, this review will determine whether the overall conclusions related to the phytochemical subgroups are the same for the identified health conditions useful to public health policymakers and health professionals alike. Review papers to date are often focused on only one phytochemical class or for meta-analyses only focus on randomised controlled trials. By considering all of the evidence for the growing research into phytochemicals, the proposed review will provide readers with a comprehensive update of food-related studies and their impact on health.

\section{Twitter Follow Yasmine Probst @YasmineProbst}

Acknowledgements The authors would also like to thank Ms Melanie Thompson for her assistance with planning the search strategy and Ms Sarah McLeod for her assistance with screening of articles.

Contributors YCP designed the study and will oversee the implementation. YCP, KK and VXG all contributed to the preparation of the manuscript.

Funding The protocol development of this review was supported by a National Health and Medical Research Council Translating Research into Practice Research Fellowship which supported the salary of the lead author in developing the protocol. At the time of the protocol publication, no funding was available to support completion of the review.

Competing interests None declared.

Provenance and peer review Not commissioned; externally peer reviewed.

Data sharing statement On completion of the review outlined in the protocol, the authors are willing to share the outcomes on request.

Open Access This is an Open Access article distributed in accordance with the Creative Commons Attribution Non Commercial (CC BY-NC 4.0) license, which permits others to distribute, remix, adapt, build upon this work noncommercially, and license their derivative works on different terms, provided the original work is properly cited and the use is non-commercial. See: http:// creativecommons.org/licenses/by-nc/4.0/

\section{REFERENCES}

1. Dillard CJ, German JB. Phytochemicals: nutraceuticals and human health. J Sci Food Agric 2000;80:1744-56.

2. Knekt $P$, Kumpulainen J, Järvinen $\mathrm{R}$, et al. Flavonoid intake and risk of chronic diseases. Am J Clin Nutr 2002;76:560-8.

3. Khalesi S, Irwin C, Schubert M. Flaxseed consumption may reduce blood pressure: a systematic review and meta-analysis of controlled trials. J Nutr 2015;145:758-65

4. Medina-Remón A, Tresserra-Rimbau A, Pons A, et al. Effects of total dietary polyphenols on plasma nitric oxide and blood pressure in a high cardiovascular risk cohort. The PREDIMED randomized trial. Nutr Metab Cardiovasc Dis 2015;25:60-7.

5. Hooper L, Kroon PA, Rimm EB, et al. Flavonoids, flavonoid-rich foods, and cardiovascular risk: a meta-analysis of randomized controlled trials. Am J Clin Nutr 2008;88:38-50.

6. Park EJ, Pezzuto JM. Flavonoids in cancer prevention. Anticancer Agents Med Chem 2012;12:836-51.

7. Wang $\mathrm{Y}$, Cui $\mathrm{R}$, Xiao $\mathrm{Y}$, et al. Effect of carotene and lycopene on the risk of prostate cancer: a systematic review and dose-response meta-analysis of observational studies. PLOS ONE 2015;10(9):e0137427.
8. Commenges $\mathrm{D}$, Scotet $\mathrm{V}$, Renaud $\mathrm{S}$, et al. Intake of flavonoids and risk of dementia. Eur J Epidemiol 2000;16:357-63.

9. Dwyer JT, Peterson JJ. Measuring flavonoid intake: need for advanced tools. Public Health Nutr 2002;5:925-30.

10. Thompson FE SA.Dietary assessment methodology —Chapter 1. Elsevier, 2001.

11. Probst Y, Guan V, Kent K. A systematic review of food composition tools used for determining dietary polyphenol intake in estimated intake studies. Food Chem.

12. Balentine DA, Dwyer JT, Erdman JW Jr, et al Recommendations on reporting requirements for flavonoids in research. Am J Clin Nutr 2015;101:1113-25.

13. Food and Agriculture Organization. FAO/INFOODS guidelines for food matching. 1.2 edn. Rome: FAO, 2012.

14. Bosetti C, Spertini L, Parpinel M, et al. Flavonoids and breast cancer risk in Italy. Cancer Epidemiol Biomarkers Prev 2005;14:805-8.

15. Witkowska AM, Zujko ME, Waśkiewicz A, et al. Comparison of Various Databases for Estimation of Dietary Polyphenol Intake in the Population of Polish Adults. Nutrients 2015;7(11):9299-308

16. Baena Ruiz R, Salinas Hernández P. Cancer chemoprevention by dietary phytochemicals: epidemiological evidence. Maturitas 2016;94:13-19

17. Chang SK, Alasalvar C, Shahidi F. Review of dried fruits: phytochemicals, antioxidant efficacies, and health benefits. J Funct Foods 2016;21:113-32.

18. Dietz BM, Hajirahimkhan A, Dunlap TL, et al. Botanicals and their bioactive phytochemicals for women's health. Pharmacol Rev 2016;68:1026-73.

19. Goya L, Martín MÁ, Sarriá B, et al. Effect of cocoa and its flavonoids on biomarkers of inflammation: studies of cell culture, animals and humans. Nutrients 2016;8:212.

20. Rodriguez-Casado A. The health potential of fruits and vegetables phytochemicals: notable examples. Crit Rev Food Sci Nutr 2016:56:1097-107.

21. Xiao J. Phytochemicals in food and nutrition. Crit Rev Food Sci Nutr 2016;56(Suppl 1):S1-3

22. Tiwari BK, Brunton NP, Brennan C. Handbook of plant food phytochemicals: sources, stability and extraction. John Wiley \& Sons, 2013.

23. Ren R, Shi C, Cao J, et al. Neuroprotective effects of a standardized flavonoid extract of safflower against neurotoxin-induced cellular and animal models of Parkinson's disease. Sci Rep 2016;6:22135.

24. Liu XH, Yu RB, Liu R et al. Association between lutein and zeaxanthin status and the risk of cataract: a meta-analysis. Nutrients 2014:6:452-65.

25. Tapsell LC. Foods and food components in the Mediterranean diet: supporting overall effects. BMC Med 2014;12:100.

26. Van Die MD, Bone KM, Williams SG, et al. Soy and soy isoflavones in prostate cancer: a systematic review and meta-analysis of randomized controlled trials. BJU Int 2014;113(5B):E119-30.

27. Vuong QV. Epidemiological evidence linking tea consumption to human health: a review. Crit Rev Food Sci Nutr 2014;54:523-36.

28. Wang ZM, Zhao D, Nie ZL, et al. Flavonol intake and stroke risk: a meta-analysis of cohort studies. Nutrition 2014;30:518-23.

29. Tresserra-Rimbau A, Rimm EB, Medina-Remón A, et al. Inverse association between habitual polyphenol intake and incidence of cardiovascular events in the PREDIMED study. Nutr Metab Cardiovasc Dis 2014:24:639-47.

30. Mink PJ, Scrafford CG, Barraj LM, et al. Flavonoid intake and cardiovascular disease mortality: a prospective study in postmenopausal women. Am J Clin Nutr 2007;85:895-909.

31. Egert S, Rimbach $\mathrm{G}$. Which sources of flavonoids: complex diets or dietary supplements? Adv Nutr 2011;2:8-14

32. Jacobs DR, Gross MD, Tapsell LC. Food synergy: an operational concept for understanding nutrition. Am J Clin Nutr 2009;89: 1543S-8S.

33. Neacsu M, McMonagle J, Fletcher RJ, et al. Availability and dose response of phytophenols from a wheat bran-rich cereal product in healthy human volunteers. Mol Nutr Food Res 2016.

34. Vitaglione P, Mennella I, Ferracane R, et al. Whole-grain wheat consumption reduces inflammation in a randomized controlled trial on overweight and obese subjects with unhealthy dietary and lifestyle behaviors: role of polyphenols bound to cereal dietary fiber. Am J Clin Nutr 2015;101:251-61.

35. Forbes-Hernandez TY, Gasparrini M, Afrin S, et al. The healthy effects of strawberry polyphenols: which strategy behind antioxidant capacity? Crit Rev Food Sci Nutr 2016;56(Suppl 1):S46-59.

36. Meigs JB, Hu FB, Rifai N, et al. Biomarkers of endothelial dysfunction and risk of type 2 diabetes mellitus. JAMA 2004;291:1978-86.

37. von Scholten BJ, Reinhard $\mathrm{H}$, Hansen TW, et al. Markers of inflammation and endothelial dysfunction are associated with 
incident cardiovascular disease, all-cause mortality, and progression of coronary calcification in type 2 diabetic patients with

microalbuminuria. J Diabetes Complications 2016;30:248-55.

38. Pradhan AD, Manson JE, Rifai N, et al. C-reactive protein, interleukin 6 , and risk of developing type 2 diabetes mellitus. JAMA 2001;286:327-34.

39. Allin KH, Bojesen SE, Nordestgaard BG. Inflammatory biomarkers and risk of cancer in 84,000 individuals from the general population. Int J Cancer 2016;139:1493-500.

40. Wang X, Ouyang YY, Liu J, et al. Flavonoid intake and risk of CVD: a systematic review and meta-analysis of prospective cohort studies. B J Nutr 2014;111:1-11.

41. Grimes DA, Schulz KF. An overview of clinical research: the lay of the land. Lancet 2002;359:57.

42. Chong MF, George TW, Alimbetov D, et al. Impact of the quantity and flavonoid content of fruits and vegetables on markers of intake in adults with an increased risk of cardiovascular disease: the FLAVURS trial. Eur J Nutr 2013;52:361-78.

43. Neuhouser ML. Review: dietary flavonoids and cancer risk: evidence from human population studies. Nutr Cancer 2004 $50: 1-7$.
44. Hayes JD, Kelleher MO, Eggleston IM. The cancer chemopreventive actions of phytochemicals derived from glucosinolates. Eur J Nutr 2008;47:73-88.

45. Wallace TC, Slavin M, Frankenfeld CL. Systematic review of anthocyanins and markers of cardiovascular disease. Nutrients 2016;8:pii: E32.

46. Li SH, Zhao P, Tian HB, et al. Effect of grape polyphenols on blood pressure: a meta-analysis of randomized controlled trials. PLoS ONE 2015;10:e0137665.

47. Zhang GQ, Chen JL, Liu Q, et al. Soy intake is associated with lower endometrial cancer risk: a systematic review and meta-analysis of observational studies. Medicine (Baltim) 2015;94:e2281.

48. Moher D, Liberati A, Tetzlaff J, et al. Preferred reporting items for systematic reviews and meta-analyses: the PRISMA statement. Int J Surg 2010;8:336-41.

49. Shamseer L, Moher D, Clarke $M$, et al. Preferred reporting items for systematic review and meta-analysis protocols (PRISMA-P) 2015: elaboration and explanation. BMJ 2015;349:g7647.

50. Higgins J, Green S, eds. Cochrane handbook for systematic reviews of interventions, version 5.1.0. updated March 2011 edn. London: The Cochrane Collaboration, 2011. 